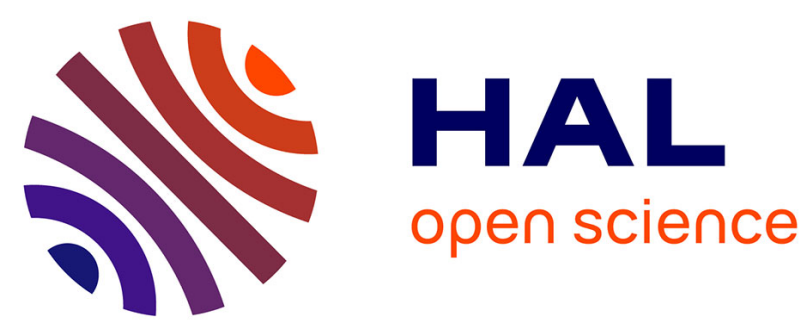

\title{
ON MULTIVARIATE NON-GAUSSIAN SCALE INVARIANCE: FRACTIONAL LÉVY PROCESSES AND WAVELET ESTIMATION
}

B Cooper Boniece, Gustavo Didier, Herwig Wendt, Patrice Abry

\section{- To cite this version:}

B Cooper Boniece, Gustavo Didier, Herwig Wendt, Patrice Abry. ON MULTIVARIATE NONGAUSSIAN SCALE INVARIANCE: FRACTIONAL LÉVY PROCESSES AND WAVELET ESTIMATION. European Signal Processing Conference (EUSIPCO), Sep 2019, A Coruna, Spain. hal02361748

\author{
HAL Id: hal-02361748 \\ https://hal.science/hal-02361748
}

Submitted on 13 Nov 2019

HAL is a multi-disciplinary open access archive for the deposit and dissemination of scientific research documents, whether they are published or not. The documents may come from teaching and research institutions in France or abroad, or from public or private research centers.
L'archive ouverte pluridisciplinaire HAL, est destinée au dépôt et à la diffusion de documents scientifiques de niveau recherche, publiés ou non, émanant des établissements d'enseignement et de recherche français ou étrangers, des laboratoires publics ou privés. 


\title{
ON MULTIVARIATE NON-GAUSSIAN SCALE INVARIANCE: FRACTIONAL LÉVY PROCESSES AND WAVELET ESTIMATION
}

\author{
B. Cooper Boniece ${ }^{(1)}$, Gustavo Didier ${ }^{(1)}$, Herwig Wendt ${ }^{(2)}$, Patrice Abry ${ }^{(3)}$ \\ (1) Tulane University, New Orleans, LA, USA \\ (2) IRIT, CNRS (UMR 5505), Université de Toulouse, France \\ (3) Univ Lyon, ENS de Lyon, Univ Claude Bernard, CNRS, Laboratoire de Physique, Lyon, France
}

\begin{abstract}
In the modern world of "Big Data," dynamic signals are often multivariate and characterized by joint scale-free dynamics (self-similarity) and non-Gaussianity. In this paper, we examine the performance of joint wavelet eigenanalysis estimation for the Hurst parameters (scaling exponents) of non-Gaussian multivariate processes. We propose a new process called operator fractional Lévy motion (ofLm) as a Lévy-type model for non-Gaussian multivariate self-similarity. Based on large size Monte Carlo simulations of bivariate ofLm with a combination of Gaussian and non-Gaussian marginals, the estimation performance for Hurst parameters is shown to be satisfactory over finite samples.
\end{abstract}

Index Terms - multivariate self-similarity, non-Gaussian process, Lévy processes, wavelets.

\section{INTRODUCTION}

Univariate self-similarity. A signal is called scale invariant when its temporal dynamics lack a characteristic scale. Under scale invariance, a continuum of time scales contributes to the observed dynamics, and the analyst's focus is on identifying mechanisms that relate the scales, often in the form of the so-called scaling exponents $[1,2,3]$. Scale invariant dynamic signals are phenomenologically observed in a wide range of physical and engineering systems. In applications, it often manifests itself in the form of self-similarity. A signal $X$ is called self-similar when its finite-dimensional distributions (fdd) are invariant with respect to a suitable scaling of time, i.e., $\{X(t)\}_{t \in \mathbb{R}} \stackrel{\text { fdd }}{=}\left\{a^{H} X(t / a)\right\}_{t \in \mathbb{R}}, a>0$, where $H$ is the so-named Hurst parameter. The celebrated fractional Brownian motion $(\mathrm{fBm})$ is the only Gaussian, self-similar process with stationary increments [4]. Estimation of the Hurst parameter $H$ is of central interest for signal processing tasks such as characterization, diagnosis, classification and detection. It is now well documented that the wavelet transform

Work supported by Grant ANR-16-CE33-0020 MultiFracs. G.D. was partially supported by the ARO grant W911NF-14-1-0475. G.D.'s long term visits to France were supported by ENS de Lyon, CNRS and the Carol Lavin Bernick faculty grant. provides efficient multiscale representations, leading to theoretically well-grounded, robust and accurate estimation of $H[2,5]$. However, success has remained mostly restricted to univariate analysis. In the modern world of "Big Data," a plethora of sensors monitor natural and artificial systems, generating large data sets in the form of several joint time series. This creates an ever-increasing demand for adequate multivariate self-similarity modeling.

Related work. Operator fractional Brownian motion (ofBm), a generalization of $\mathrm{fBm}$, was recently put forward as a model for multivariate Gaussian self-similarity $[6,7,8$, 9]. In particular, it allows multiple correlated fBm coordinate processes with possibly distinct self-similarity exponents $H_{m}, m=1, \ldots, M$, occurring in a non-canonical set of coordinates (mixing). In $[10,11]$, a statistical method is devised for jointly estimating the vector of self-similarity exponents $\underline{H}=\left(H_{1}, \ldots, H_{M}\right)$. Based on multivariate wavelet representations and eigenvalue decompositions, the method was mathematically studied and shown to have satisfactory theoretical and practical performance.

Furthermore, non-Gaussian behavior is pervasive in a myriad of natural phenomena (e.g., turbulence, anomalous diffusion $[11,12])$ and artificial systems (e.g., Internet traffic [13]). Among non-Gaussian scale invariant models, fractional Lévy processes (e.g., [14, 15, 16, 17, 18]) have become popular in physical applications since they make up a very broad family of second order models displaying fractional covariance structure [19, 20, 21, 22, 23]. Nevertheless, the modeling of non-Gaussian, multivariate fractional Lévy signals, while of great importance in applications, is a research topic that has been relatively little explored (e.g., [24, 25, 26]), and the estimation of scaling exponent even less.

Goals, contributions and outline. In this paper, we examine the performance of joint wavelet eigenanalysis estimation for non-Gaussian multivariate scaling processes. To this end, we define a new class of multivariate non-Gaussian fractional stochastic processes called operator fractional Lévy motion (ofLm) (Section 2). This class encompasses and generalizes the Gaussian class of ofBm, while conveniently preserving the property of covariance (operator) self-similarity. After in- 
troducing wavelet eigenanalysis estimation (Section 3), performance is then assessed with respect to non-Gaussianity by means of Monte Carlo experiments (Section 4). To illustrate the versatility of the method and the effect of non-Gaussian marginal distributions, we allow the unobserved signal to contain both non-Gaussian and Gaussian entries.

\section{OPERATOR FRACTIONAL LÉVY MOTION}

Operator self-similarity. Let $\underline{H}=P \operatorname{diag}(\underline{H}) P^{-1}$ be the so-named Hurst matrix parameter, where the vector $\underline{H}$ contains the Hurst exponents (eigenvalues). For a given process $Y=\left(Y_{1}, \ldots, Y_{n}\right)$ with Hurst matrix parameter $\underline{H}$, multivariate covariance self-similarity, a key property of ofBm and ofLm, reads as

$$
\mathbb{E} Y(s) Y(t)^{*}=a \underline{\underline{H}} \mathbb{E} Y(s / a) Y(t / a)^{*} a \underline{\underline{H}}^{*},
$$

for all $a>0$, where $a \underline{\underline{H}}:=\sum_{k=0}^{+\infty} \log ^{k}(a) \underline{\underline{H}}^{k} / k$ !. When the mixing matrix $P$ is diagonal, namely, when we can set $P \equiv$ $I$, the covariance self-similarity relation (1) takes the simple form of component-wise covariance self-similarity relations

$$
\mathbb{E} Y_{\ell}(s) Y_{\ell^{\prime}}(t)=a^{H_{\ell}+H_{\ell^{\prime}}} \mathbb{E} Y_{\ell}(s / a) Y_{\ell^{\prime}}(t / a),
$$

for $a>0$ and $\ell, \ell^{\prime}=1,2, \ldots, n$.

\subsection{Operator fractional Lévy motion}

OfLm is a new and versatile class of non-Gaussian multivariate fractional processes displaying the same covariance structure of ofBm. For clarity of exposition and without loss of generality, we construct ofLm in the bivariate case. Let $\left\{L(t)=\left(L_{1}(t), L_{2}(t)\right)\right\}_{t \in \mathbb{R}}$ be a two-sided symmetric Lévy process in $\mathbb{R}^{2}$ with $\mathbb{E} L(1) L(1)^{*}=: \Sigma_{L},\left|\Sigma_{L}\right|<\infty$ (e.g., [27]). Let $0<H_{1} \leq H_{2}<1$, and define the (pre-mixed) process $X$ by the component-wise convolution $X(t)=\left(g_{t} * \dot{L}\right)(t)$, where $g$ is the diagonal-matrix-valued fractional kernel $g_{t}(u):=u_{+}^{D}-(u-t)_{+}^{D}, D=\operatorname{diag}(\underline{H})-\frac{1}{2} I$. Then, the entrywise processes $X_{H_{\ell}}, \ell=1,2$, are generally correlated fractional Lévy processes with corresponding Hurst parameters $H_{\ell} \in(0,1)$ (e.g., $\left.[14,16]\right)$. In particular, each has stationary increments and its covariance function is identical to that of $\mathrm{fBm}$, i.e.,

$$
\mathbb{E} X_{H_{\ell}}(t) X_{H_{\ell}}(s)=\left\{|t|^{2 H_{\ell}}+|s|^{2 H_{\ell}}-|t-s|^{2 H_{\ell}}\right\} \sigma_{\ell}^{2} / 2 .
$$

Note that, if non-Gaussian, the stochastic behavior of the process $X_{H_{\ell}}$ is not characterized by its covariance function. Let $P$ be a $2 \times 2$, real-valued, invertible matrix. We define (bivariate) ofLm $Y \underline{H}, L, P$ by

$$
\left\{Y_{1}^{\underline{H}, L, P}(t), Y_{2}^{\underline{H}, L, P}(t)\right\}_{t \in \mathbb{R}}=P\left\{X_{H_{1}}(t), X_{H_{2}}(t)\right\}_{t \in \mathbb{R}}
$$

(in short, $Y \underline{H}, L, P=P X)$. Bivariate ofLm is welldefined only if $\Gamma\left(2 H_{1}+1\right) \Gamma\left(2 H_{2}+1\right) \sin \left(\pi H_{1}\right) \sin \left(\pi H_{2}\right)$ $-\rho^{2} \Gamma\left(H_{1}+H_{2}+1\right)^{2} \sin ^{2}\left(\pi\left(H_{1}+H_{2}\right) / 2\right)>0$, where $\rho:=\operatorname{Corr}\left(X_{H_{1}}(1), X_{H_{2}}(1)\right)$, thus showing that $\underline{H}$ and $\rho$ cannot be selected independently (cf. [8]). The process $Y \underline{H}, L, P$ exhibits the operator covariance self-similarity property (1).

\section{WAVELET EIGENVALUE-BASED ESTIMATION}

In the statistical analysis of self-similar signals, the central task is to estimate the Hurst exponents $\underline{H}=\left(H_{1}, H_{2}\right)$ from a single time series $Y$. When $P$ is diagonal, (2) suggests that $H_{1}$ and $H_{2}$ can be estimated independently using standard univariate methodologies [28, 29]. However, in the general framework of nondiagonal mixing (coordinates) matrices $P$, univariate estimation does not yield relevant results. For this reason, we put forward a multivariate wavelet transformbased joint estimation methodology that has been successfully applied to the Gaussian case (cf. [10,11]) .

Multivariate wavelet transform. Let $\psi_{0}$ be a mother wavelet, i.e., a reference oscillating pattern (function) with good joint time and frequency localization. A mother wavelet is parametrized by the so-named number of vanishing moments $N_{\psi}$, i.e., a positive integer such that $\forall n=0, \ldots, N_{\psi}-$ $1, \int_{\mathbb{R}} t^{n} \psi_{0}(t) d t \equiv 0$ and $\int_{\mathbb{R}} t^{N_{\psi}} \psi_{0}(t) d t \neq 0$. Let $\left\{\psi_{j, k}(t)=\right.$ $\left.2^{-j / 2} \psi_{0}\left(2^{-j} t-k\right)\right\}_{(j, k) \in \mathbb{N}^{2}}$ be the collection of dilated and translated templates of $\psi_{0}$ that forms an orthonormal basis of $\mathcal{L}^{2}(\mathbb{R})$.

The multivariate discrete wavelet transform (DWT) of the multivariate stochastic process $\{Y(t)\}_{t \in \mathbb{R}}$ is defined as $\left(D\left(2^{j}, k\right)\right) \equiv D_{Y}\left(2^{j}, k\right)=\left(D_{Y_{1}}\left(2^{j}, k\right), D_{Y_{2}}\left(2^{j}, k\right)\right), \forall k \in$ $\mathbb{Z}, \forall j \in\left\{j_{1}, \ldots, j_{2}\right\}$, and $\forall m \in\{1,2\}: D_{Y_{m}}\left(2^{j}, k\right)=$ $\left\langle 2^{-j / 2} \psi_{0}\left(2^{-j} t-k\right) \mid Y_{m}(t)\right\rangle$.

Joint estimation of $\boldsymbol{H}_{\mathbf{1}}, \boldsymbol{H}_{\mathbf{2}}$. Let $S\left(2^{j}\right)$ denote the empirical wavelet spectrum, defined as

$$
S\left(2^{j}\right)=\frac{1}{n_{j}} \sum_{k=1}^{n_{j}} D\left(2^{j}, k\right) D\left(2^{j}, k\right)^{*}, \quad n_{j}=\frac{N}{2^{j}},
$$

where $N$ is the sample size. Let $\Lambda\left(2^{j}\right)=\left\{\lambda_{1}\left(2^{j}\right), \lambda_{2}\left(2^{j}\right)\right\}$ be the eigenvalues of the $2 \times 2$ matrix $S\left(2^{j}\right)$. We define the wavelet eigenvalue regression estimators $\left(\widehat{H}_{1}, \widehat{H}_{2}\right)$ of $\left(H_{1}, H_{2}\right)$ by means of weighted log-regressions across scales $2^{j_{1}} \leq a \leq 2^{j_{2}}$

$$
\widehat{H}_{m}=\left(\sum_{j=j_{1}}^{j_{2}} w_{j} \log _{2} \lambda_{m}\left(2^{j}\right)\right) / 2-\frac{1}{2}, \quad \forall m=1,2 .
$$

For purely Gaussian instances (ofBm), it was shown theoretically in $[10,11]$ that $\left(\widehat{H}_{1}, \widehat{H}_{2}\right)$ are consistent estimators with asymptotic joint normality under mild assumptions. Also, these estimators have very satisfactory performance for finite sample sizes. Their covariances decrease as a function of the inverse of the sample size and are approximately normal even for small sample sizes. It was further observed that the variances of $\left(\widehat{H}_{1}, \widehat{H}_{2}\right)$ do not significantly depend on the actual values of $\left(H_{1}, H_{2}\right)$.

\section{ESTIMATION PERFORMANCE ASSESSMENT}

With a view towards contrasting the tail behavior of ofLm to its purely Gaussian counterpart, ofBm, we conducted Monte 
Carlo studies for the case where the non-Gaussian part of $L_{1}$ is symmetric tempered stable, i.e., the marginals of $X_{H_{1}}$ display distinctly non-Gaussian tail behavior but have finite moments of all orders [30, 31]. Such processes are parameterized by a stability index $\alpha \in(0,2)$ and a tempering parameter $\gamma>0$, and can be constructed by exponentially tempering the density $p(x, t)$ at time $t$ of an $\alpha$-stable process: $p_{\gamma}(x, t) \propto e^{-\gamma|x|} p(x, t)$ [31]; increasingly small tempering parameter $\gamma$ corresponds to increasingly heavier-tailed behavior. For comparison, we take $L_{2}$ to be Gaussian (i.e., $X_{H_{2}}$ is an $\mathrm{fBm}$ ), so the resulting bivariate ofLm $Y \underline{H}, L, P$ contains a combination (sum) of both Gaussian and non-Gaussian components.

\subsection{Monte-Carlo simulation}

In all instances, we generated sets of independent ofLm paths at the sample size $n=2^{15}$, based on a combination of fractional Lévy process [16] and fBm with corresponding Hurst exponents $H_{1}=0.35$ and $H_{2}=0.75$, respectively. The tempered stable part of $L_{1}$ was chosen with $\alpha=1$ and different $\gamma$ for each individual study (described below); the Gaussian part of $L_{1}$ was chosen as the same as $L_{2}$. Consequently, $\Sigma_{L}$ is non-diagonal and $X_{H_{1}}$ and $X_{H_{2}}$ are correlated, i.e., $\mathbb{E} X_{H_{1}}(t) X_{H_{2}}(s)={ }_{c} \int_{\mathbb{R}} g_{H_{1}, t}(x) g_{H_{2}, s}(x) d x$, where $g_{H_{\ell}, u}(x):=(u-x)_{+}^{H_{\ell}-1 / 2}-(-x)_{+}^{H_{\ell}-1 / 2}$. The $\mathrm{fBm}$ was simulated via circulant matrix embedding (e.g., [32]). The symmetric tempered stable marginals were simulated via an accept-reject procedure for the tempered stable random variables and an adaptation of the FFT-based algorithm described in [33] for the tempered case. In the notation of [33], the mesh size was chosen $m=8$ with kernel cutoff parameter $M=2^{15}$. The mixing matrix was set to $P=\left((1,0)^{T} ;(1,1)^{T}\right)$. For this choice, it can be shown that $Y_{1}^{\underline{H}, L, P}$ is a sum of Gaussian and non-Gaussian components, and that $Y_{2}^{\underline{H}}, L, P$ is Gaussian.

Computational studies were carried out as follows. In all instances, Daubechies wavelets with $N_{\psi}=2$ vanishing moments were used. In Figure $1, R=1000$ independent ofLm paths were generated, and both component-wise (univariate) wavelet regression and wavelet eigenvalue regression were conducted over the octave range $\left(j_{1}, j_{2}\right)=\left(4, \log _{2} n-\right.$ $\left.N_{\psi}-2\right)=(4,11)$. In Figure 2, the wavelet eigenvalue regression was carried out over $\left(j_{1}, j_{2}\right)=(4,11)$ for seven sets of $R=1000$ paths with $\gamma \in\left\{10^{-7}, 10^{-6}, \ldots, 10^{-1}\right\}$, and the estimates from these paths were used in Figure 3 for $\gamma \in\left\{10^{-6}, \ldots, 10^{-3}\right\}$. In Figure 4, two sets of $R=1000$ ofLm paths were generated with $\gamma \in\left\{10^{-5}, 10^{-2}\right\}$ and were analyzed using the octave range $\left(j_{1}, j_{2}\right)=(3+k, 7+k)$ for $k=0,1, \ldots, 5$, and another set of $R=1000$ ofBm paths was generated and analyzed over the same octave ranges for comparison.

Performance with respect to non-Gaussianity. Figure 1 shows that univariate-like (component-wise) wavelet analysis
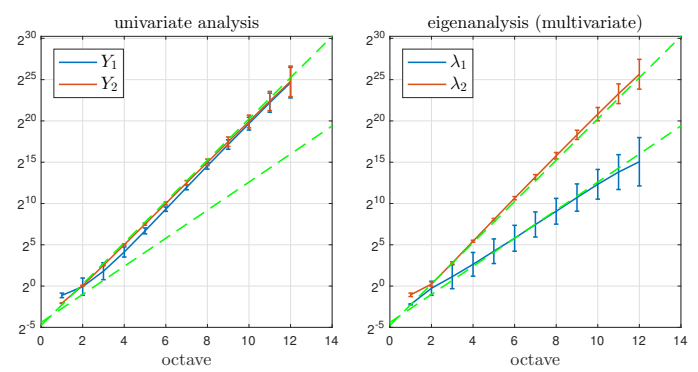

Fig. 1. Monte Carlo wavelet log-scaling plots. Average curves for component-wise (univariate) wavelet scaling plots (left) and wavelet eigenvalue scaling plots $\log \lambda_{1}\left(2^{j}\right), \log \lambda_{2}\left(2^{j}\right)$ (right) over $R=1000$ runs for the ofLm with $\gamma=10^{-5}$. The error bars represent approximate $95 \%$ confidence intervals; the green dashed reference lines are the theoretical slopes associated with $H_{1}$ and $H_{2}$. The multivariate analysis manages to identify the presence of a smaller Hurst exponent $H_{1}<H_{2}$ as well as that of distinctly non-Gaussian/heavier-tailed behavior (larger standard error around the $\lambda_{1}$ log-scaling curve). Both phenomena are missed by univariate-like analysis.

is overborne by the dominant exponent $H_{2}>H_{1}$. It detects neither the presence of the smaller Hurst exponent $H_{1}$ nor of non-Gaussian tail behavior. By contrast, multivariate analysis reveals the presence of both exponents $H_{1}$ and $H_{2}$ as well as that of non-Gaussian tail behavior. In all results from multivariate analysis, the effect of the presence of non-Gaussianity turned out more strongly in the scaling behavior of the smaller eigenvalue $\lambda_{1}\left(2^{j}\right)$. In other words, not only is $\lambda_{1}\left(2^{j}\right)$ driven by the smaller Hurst exponent $H_{1}$, as typical in wavelet eigenanalysis, but also the non-Gaussianity of the pre-mixed component $X_{H_{1}}$, associated with $H_{1}$, was mostly absorbed by $\lambda_{1}\left(2^{j}\right)$. Accordingly, the scaling of the larger eigenvalue $\lambda_{2}\left(2^{j}\right)$ is, over large scales, driven by $H_{2}$ and similar to that for the wavelet eigenanalysis of (the purely Gaussian) ofBm. By construction, the wavelet (second order) covariance structure of ofLm is identical to that of an ofBm. However, the confidence intervals associated with the scaling curves of $\lambda_{1}\left(2^{j}\right)$ behave differently compared to the purely Gaussian case, since such intervals involve fourth order properties of the underlying process. In particular, whereas wavelet scaling curves display increased variability for larger octaves in the purely Gaussian case, it is a striking feature of ofLm that confidence intervals associated with $\lambda_{1}\left(2^{j}\right)$ are shown in our studies to be approximately constant as a function of $j$ for moderate $j$. Other choices of $P$ yielded qualitatively similar results; for most choices of $P$, the large-variance behavior associated with non-Gaussianity generally displays in both eigenvalues.

In more detail, Figure 2 shows that the wavelet eigenvalue regression bias remains approximately fixed as a function of $\gamma$, and is comparable to that observed for ofBm instances. However, the estimation performance degrades for small $\gamma$ 

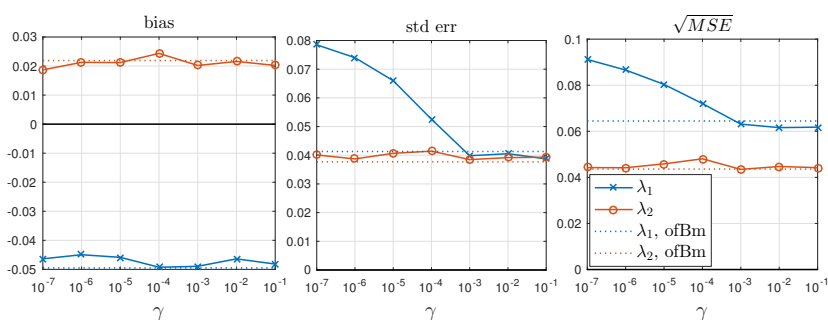

Fig. 2. Estimation performance over different tail behavior. Bias, standard error, and $\sqrt{M S E}$ as the tempering parameter $\gamma$ increases (i.e., increasingly lighter tails). Compared to ofBm (dashed reference lines), the presence of heaviertailed noise has little effect on the bias. However, for standard error, performance degrades significantly for smaller $\gamma$.

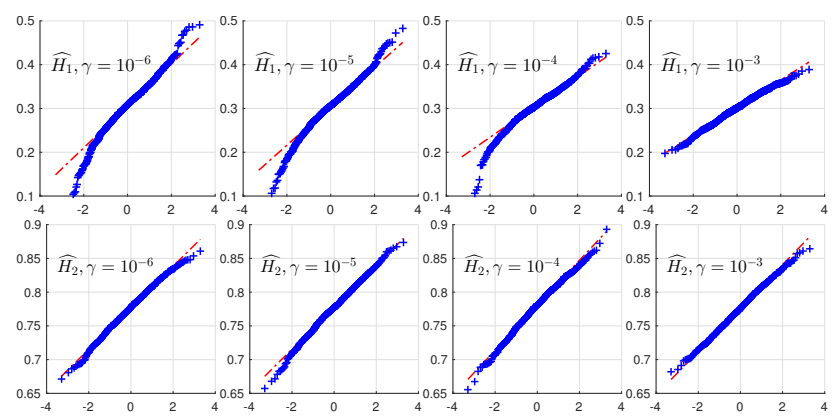

Fig. 3. Non-Gaussianity of estimates. Q-Q plots for $\widehat{H}_{\ell}$, $\ell=1,2$ for $\gamma \in\left\{10^{-6}, \ldots, 10^{-3}\right\}$. The distribution of $\widehat{H}_{2}$ is approximately Gaussian for all $\gamma$. In contrast, as the Lévy noise tail behavior departs further from Gaussianity, $\widehat{H}_{1}$ appears increasingly non-Gaussian, i.e., the asymptotically Gaussian behavior of the estimator $\widehat{H}_{1}$ requires larger sample sizes to manifest itself.

due to the presence of heavier tails. Interestingly, among the subclass of ofLms with tempered stable driving Lévy noise, all studies conducted suggest that the estimation performance degrades only in standard error as a function of the tail behavior of the driving process. This indicates that the wavelet eigenvalue regression method is bias-robust with respect to non-Gaussianity.

In Figure 3, at the sample size $n=2^{15}$, asymptotic Gaussianity is achieved for $\widehat{H}_{2}$ over all instances $\gamma \in$ $\left\{10^{-6}, \ldots, 10^{-3}\right\}$. However, in the presence of heaviertailed behavior, $\widehat{H}_{1}$ departs further from Gaussianity due to the increased variability of the eigenvalue curve $\lambda_{1}\left(2^{j}\right)$, indicating that larger sample sizes are required to achieve Gaussian behavior for $\widehat{H}_{1}$ when $\gamma$ is small.

Performance with respect to scale choices. In Figure 4 , the optimal choice of regression octave range $\left(j_{1}, j_{2}\right)$ for ofLm is similar to that for ofBm. Compared to the case for ofBm, a similar bias-variance tradeoff occurs: the eigenvalues $\lambda_{1}\left(2^{j}\right), \lambda_{2}\left(2^{j}\right)$ display asymptotic scaling according to $H_{1}$ and $H_{2}$, indicating that larger octave values $j_{1}, j_{2}$ tend to minimize bias, to a point; however, for large $j$, they also exhibit larger variance due to the fewer number of terms $n_{j}$ in the associated wavelet matrices $S\left(2^{j}\right)$ (cf. Figure 1), as with ofBm. In addition, when $\gamma$ is large (lighter tails), the performance of wavelet eigenvalue regression estimation is close to that for an ofBm, resulting in the same choice of optimal $\left(j_{1}, j_{2}\right)$. When $\gamma$ is small (heavier tails), the performance degrades in standard error. However, the resulting standard error curve associated with $\widehat{H}_{1}$ is approximately a translation of the curve corresponding to the ofBm case. This leads to reduced $\sqrt{M S E}$ performance, but also to the same choice of optimal $\left(j_{1}, j_{2}\right)$ as compared to ofBm.
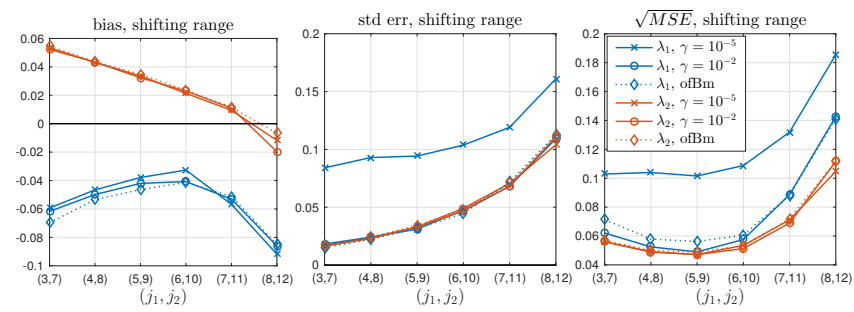

Fig. 4. Choice of regression range over small $\left(10^{-5}\right)$ and large $\left(10^{-2}\right)$ values of $\gamma$. Bias, standard error, and $\sqrt{M S E}$ as the regression range $\left[j_{1}, j_{2}\right]$ shifts from $[3,7]$ to $[8,12]$. For comparison, the dashed lines represent results for purely Gaussian (ofBm) instances. When $\gamma$ is small (heavier tails), the increased variability in the wavelet eigenvalues $\lambda_{1}\left(2^{j}\right)$ gives rise to increased variability in the estimates $\widehat{H}_{1}$; the optimal choice of scales is the same for both heavy and lightertailed cases.

\section{CONCLUSION}

In this work, we propose a new model for non-Gaussian scaling dynamics called operator fractional Lévy motion (ofLm). Given the distributions of the driving noise components, ofLm is parametrized by a vector of Hurst (scaling exponent) parameters and a coordinates (mixing) matrix $P$. We further construct a wavelet eigenanalysis-based method for the identification of ofLm. For bivariate instances based on a combination of Gaussian and non-Gaussian marginals, large size Monte Carlo studies demonstrate that the estimation of Hurst parameters is satisfactory. We further characterize the effect of non-Gaussian marginals on wavelet-based estimation as a function of the strength of the distribution tail tempering parameter. In particular, unlike univariate-type analysis, we show that the proposed multivariate analysis method is able to identify the presence of multiple Hurst exponents as well as that of non-Gaussian noise.

Future work includes mathematically establishing the properties of the proposed wavelet estimation procedure as well as the probabilistic characterization of ofLm. The combined ofLm-wavelets framework will further be used in applications such as in neuroscientific and Internet traffic data, which are known to display conspicuous non-Gaussian traits that can be naturally modeled by means of Lévy-type processes. 


\section{REFERENCES}

[1] G. Wornell and A. Oppenheim, "Estimation of fractal signals from noisy measurements using wavelets," IEEE Transactions on Signal Processing, vol. 40, no. 3, pp. 611-623, 1992.

[2] P. Flandrin, "Wavelet analysis and synthesis of fractional Brownian motion," IEEE Transactions on Information Theory, vol. 38, pp. 910 - 917, March 1992.

[3] D. Veitch and P. Abry, "A wavelet-based joint estimator of the parameters of long-range dependence," IEEE Trans. Inform. Theory, vol. 45, no. 3, pp. 878-897, 1999.

[4] M. S. Taqqu, "Fractional Brownian motion and long range dependence," in Theory and Applications of Long-Range Dependence (P. Doukhan, G. Oppenheim and M. S. Taqqu, eds.), pp. 5-38. Birkhäuser, Boston, 2003.

[5] D. Veitch and P. Abry, "A wavelet-based joint estimator of the parameters of long-range dependence," IEEE Transactions on Information Theory, vol. 45, no. 3, pp. 878-897, April 1999.

[6] M. Maejima and J. Mason, "Operator-self-similar stable processes," Stochastic Processes and their Applications, vol. 54, pp. 139-163, 1994.

[7] J. D. Mason and Y. Xiao, "Sample path properties of operatorself-similar Gaussian random fields," Theory of Probability and its Applications, vol. 46, no. 1, pp. 58-78, 2002.

[8] G. Didier and V. Pipiras, "Integral representations and properties of operator fractional Brownian motions," Bernoulli, vol. 17, no. 1, pp. 1-33, 2011.

[9] G. Didier and V. Pipiras, "Exponents, symmetry groups and classification of operator fractional Brownian motions," Journal of Theoretical Probability, vol. 25, pp. 353-395, 2012.

[10] P. Abry and G. Didier, "Wavelet estimation for operator fractional Brownian motion," Bernoulli, vol. 24, no. 2, pp. 895928, 2018.

[11] P. Abry and G. Didier, "Wavelet eigenvalue regression for $n$ variate operator fractional Brownian motion," Journal of Multivariate Analysis, vol. 168, pp. 75-104, November 2018.

[12] B. C. Boniece, G. Didier, and F. Sabzikar, "On fractional Lévy processes: tempering, sample path properties and stochastic integration," Preprint, pp. 1-46, 2018.

[13] W. Willinger, R. Govindan, S. Jamin, V. Paxson, and S. Shenker, "Scaling phenomena in the Internet: critically examining criticality," Proceedings of the National Academy of Sciences, vol. 99, no. suppl. 1, pp. 2573-2580, 2002.

[14] A. Benassi, S. Cohen, and J. Istas, "Identification and properties of real harmonizable fractional Lévy motions," Bernoulli, vol. 8, no. 1, pp. 97-115, 2002.

[15] P. J. Brockwell and T. Marquardt, "Lévy-driven and fractionally integrated ARMA processes with continuous time parameter," Statistica Sinica, pp. 477-494, 2005.

[16] T. Marquardt, "Fractional Lévy processes with an application to long memory moving average processes," Bernoulli, vol. 12, no. 6, pp. 1099-1126, 2006.
[17] C. Lacaux and J.-M. Loubes, "Hurst exponent estimation of fractional Lévy motion," ALEA: Latin American Journal of Probability and Mathematical Statistics, vol. 3, pp. 143-164, 2007.

[18] C. Bender and T. Marquardt, "Stochastic calculus for convoluted Lévy processes," Bernoulli, vol. 14, no. 2, pp. 499-518, 2008.

[19] O. Barndorff-Nielsen and J. Schmiegel, "Time change, volatility, and turbulence," in Mathematical Control Theory and Finance, pp. 29-53. Springer, 2008.

[20] N. Suciu, "Spatially inhomogeneous transition probabilities as memory effects for diffusion in statistically homogeneous random velocity fields," Physical Review E, vol. 81, no. 5, pp. 056301, 2010.

[21] M. Magdziarz and A. Weron, "Ergodic properties of anomalous diffusion processes," Annals of Physics, vol. 326, no. 9, pp. 2431-2443, 2011.

[22] S. Zhang, Z. Lin, and X. Zhang, "A least squares estimator for Lévy-driven moving averages based on discrete time observations," Communications in Statistics-Theory and Methods, vol. 44, no. 6, pp. 1111-1129, 2015.

[23] Y. Xu, Y. Li, H. Zhang, X. Li, and J. Kurths, "The switch in a genetic toggle system with Lévy noise," Scientific Reports, vol. 6 , pp. 31505, 2016.

[24] T. Marquardt, "Multivariate fractionally integrated CARMA processes," Journal of Multivariate Analysis, vol. 98, no. 9, pp. 1705 - 1725, 2007.

[25] O. Barndorff-Nielsen and R. Stelzer, "Multivariate supOU processes," Annals of Applied Probability, vol. 21, no. 1, pp. 140182, 2011.

[26] M. Moser and R. Stelzer, "Functional regular variation of Lévy-driven multivariate mixed moving average processes," Extremes, vol. 16, no. 3, pp. 351-382, 2013.

[27] K. Sato, Lévy Processes and Infinitely Divisible Distributions, Cambridge University Press, U.K., 1999.

[28] H. Wendt, G. Didier, S. Combrexelle, and P. Abry, "Multivariate Hadamard self-similarity: testing fractal connectivity," Physica D: Nonlinear Phenomena, vol. 356, pp. 1-36, 2017.

[29] P.-O. Amblard and J.-F. Coeurjolly, "Identification of the multivariate fractional Brownian motion," IEEE Transactions on Signal Processing, vol. 59, no. 11, pp. 5152-5168, 2011.

[30] D. Applebaum, Lévy Processes and Stochastic Calculus, Cambridge University Press, U.K., 2009.

[31] B. Baeumer and M. M. Meerschaert, "Tempered stable Lévy motion and transient super-diffusion," Journal of Computational and Applied Mathematics, vol. 233, no. 10, pp. 24382448, 2010.

[32] R. B. Davies and D. S. Harte, "Tests for Hurst effect," Biometrika, vol. 74, no. 1, pp. 95-101, 1987.

[33] S. Stoev and M. S. Taqqu, "Simulation methods for linear fractional stable motion and FARIMA using the Fast Fourier Transform," Fractals, vol. 12, no. 1, pp. 95-121, 2004. 